\title{
Revista de Oncología keeps improving: the facts, the dream
}

Revista de Oncología gets into its fifth year. Since its launching there has been a great collective and interdisciplinary effort to achieve what it is today, the most important and most regarded scientific journal in oncology within the Spanish and the Iberoamerican oncologists. The clues for this success is based on the premises that have been implemented rigorously since the first day. It is now a good occasion to present the results of our success in this short but very active period of time and our confidence for an even better future.

Revista de Oncología is a peer reviewed journal that publishes Reviews, Original studies and Case reports in any area of oncology, including basic or experimental oncology, cancer epidemiology, and clinical oncology. It also represents an excellent tool for communication among physicians, basic. researchers and other health professionals working in the different fields of cancer

Revista de Oncología is the official publication of the Federación de Sociedades Españolas de Oncología (FESEO) (Spanish Federation of Cancer Societies, which holds together more than 1,700 members), and the Instituto Nacional de Cancerología de México (INCAN) (Mexican National Cancer Institute, that holds 600 members). Revista de Oncología has an independent governing body, the Editorial Board, appointed by FESEO and INCAN, that is responsible for all decisions taken regarding the scientific content of the journal. The Editorial Board has an Executive Committee made out of experts in the following fields: experimental/basic oncology, medical oncology, pediatric oncology, pathology, radiotherapy, statistics, and surgical oncology. The Executive Committee meets as frequently as needed to decide on acceptance of the manuscripts that will compose each issue. This governing body is supported by a panel of experts from all over the world, the Associate Editors, who provide advice on the critical decisions on the journal. Finally, the Executive Committee gets the support on daily basis from a panel of more than 200 experts in all areas of oncology, the Editorial Consultants. We are deeply grateful to them, since they are mostly responsible for the degree of seriousness and the quality achieved by this Journal.
All reviews, articles or case reports, either requested by the Editorial Board (reviews) or submitted for publication to the journal, are evaluated by two independent experts in the same field or closely related to the topic of the article. All referees are asked beforehand whether they will be willing to review and provide a written evaluation of the article. If they agree, the manuscript is sent along with a clear and complete questionnaire to address all relevant aspects of the manuscript including: originality, accuracy, methodology, accuracy and appropriateness of the references, clarity of presentation, and adequacy for the journal. Finally, the referees are asked whether the manuscript should be accepted, revised or rejected.

If both referees provide a similar decision on an article, the Executive Committee dictates a final decision based on their suggestions. However, when both referees disagree and provide opposite recommendations, the manuscript is submitted for the consideration of a third external referee. All manuscripts that received a positive evaluation by external experts, are also evaluated by professionals to secure its statistical accuracy, that appropriate methodology is used, and that it is correctly presented. At this stage, manuscripts are further evaluated by a member of the Executive Committee, and a final decision is made based on all these evaluations. The acceptance implies also that the manuscript is within the editorial policy of the journal and that all terms of good clinical and experimental practice are followed. This is a standard procedure in all high quality journals and assures high-quality manuscripts to Revista de Oncología.

The results of this policy has generated some interesting data that we are sure our actual or potential authors will be happy to know. First, Revista de Oncologia published almost equally manuscripts in Spanish and English. Thus, in 1999, 39\% of the articles published were in English. This figure raised to 53\% in 2000 and 2001, and up to 54\% in 2002. Second, our acceptance and rejection rates are in average $45 \%$ rejection for original manuscripts and $35 \%$ rejection for case reports in 2001. This figure raised to 58\% rejection for original manuscripts in 2002. Overall, in 2001 there was a total rejection rate of $40 \%$ of original ma- 
nuscripts and case reports, while in 2002 this figure was of $50 \%$. This is an indication of the high standard established by the Editorial Board along these years, and the successful transmission of this message to the Editorial Consultants.

It is important also to report on the time required for handling of the manuscripts since reception until their publication. In 2002, original manuscripts took an average of 116 days (3.8 months) from reception to final acceptance; this process included the review process, communication to authors, modifications by authors if required, and final response from the authors. We hope to be able to reduce the time for acceptance and publication with a modernisation of our on-line submission system and follow-up.

Another indication of the good health that Revista de Oncología enjoys is the history of the journal. It started as the official publication of FESEO in January 1999; on January 2001 it merged with the official journal of the INCAN and became also its official publication. Thus, Revista de Oncología became a truly international journal with two headquarters located in Spain and México, and has become the official publication of more that 2300 health professionals working on different fields of oncology. Furthermore, our journal has special links to many Iberoamerican Institutions such as SMEO, GETLAC, ALAIC, UICC, FLASCA, CRILA and many Societies and Research Centers in Central and South America.

Also it is important to take into account that the journal started as a bi-monthly publication in 1999, with six issues per year (335 pages). However, because of the increased reception of manuscripts, starting on 2002 it was necessary to increase the number of issues to nine (523 pages). As stated earlier, this has been achieved together with an increase in the rejection rate from $40 \%$ in 2001 to $50 \%$ in 2002 for original contributions. These figures speak by themselves and need no further explanation, but clearly represents the strong basis for our confidence in the future of this journal.

Finally, in the year 2000, Revista de Oncología incorporated a Teaching Section with chapters dedicated to analyse and update the basic principles from different disciplines on cancer. These chapters are written by well-known specialists in a clear and easy-to-understand language, and attempt to improve the communication among scientists and clinicians working in all fields of oncology. The first one was a course in Molecular Biology, followed by a course in Radiotherapeutic Oncology. In 2003, we are incorporating a course in Surgical Oncology. This effort is with the commitment to incorporate a multidisciplinary approach of our journal to all the oncological community. A new initiative will start in 2003 dedicated to highlight some of the most relevant papers in oncology published in other specialised journals. With this new information we hope to contribute to summarise the expanding literature on cancer to our readership.

In summary, this report is aimed to express our absolute confidence on the future of Revista de Oncologia. And for that reason, we will request the evaluation of Revista de Oncología by the Literature Selection and Technical Review Committee of the National Library of Medicine. We hope it will qualify to be accepted in the Index Medicus/Medline. This was our commitment with FESEO and INCAN. Our results indicate that this goal could now be achieved thanks to the effort and commitment of many scientists and clinicians that have supported Revista de Oncología for the last four years. There is every reason to believe that this journal will continue receiving the confidence and support of this large community in this critical year.

Juan Carlos Lacal and Alejandro Mohar Executive Editors. 